\title{
Bridging Energetics and Dynamics of Exciton Trapping in Core-Shell Quantum Dots
}

\author{
Marcello Righetto $^{+}$, Alessandro Minotto ${ }^{+}$and Renato Bozio ${ }^{+, *}$ \\ ${ }^{+}$Department of Chemical Science and U.R. INSTM, University of Padova, Via Marzolo 1, I- \\ 35131, Padova (Italy)
}

Corresponding Author: renato.bozio@unipd.it

\begin{abstract}
The widespread application of quantum dots greatly profits from their broad absorption band. However, the variable nature of excitations within these bands is expected to result in undesired excitation energy dependence of steady state emission properties. We demonstrate the different role played by hot and cold carrier trapping in determining fluorescence quantum yields. Our analysis relates the energetic parameters with the available knowledge on the dynamics of charge trapping. It turns out that de-trapping processes play a pivotal role in determining steady state emission properties. We studied excitation dependent photoluminescence quantum yields (PLQY) in different $\mathrm{CdSe} / \mathrm{Cd}_{\mathrm{x}} \mathrm{Zn}_{1-\mathrm{x}} \mathrm{S}(\mathrm{x}=0,0.5,1)$ quantum dots to identify best performing heterostructures, in terms of shell thickness and composition. Our rationalization of the observed behavior is focused on the modulation of trapping and de-trapping rates. The combination of experimental results and PLQY kinetics modeling reveals the need to consider hot-carrier trapping, supporting recent
\end{abstract}


dynamics observations. This work provides a deeper insight into trapping process in quantum dots, relating its energetics and dynamics.

\section{Introduction}

Semiconductor quantum dots (QDs) offer a wealth of opportunities for applications in many and diverse fields of biomedicine, energy and information technologies. ${ }^{1-6}$ More recently, they have expanded very fast towards the light emitting markets, namely those of display and lighting technologies. ${ }^{7}$ Their employment as down-converters in light-emitting devices (LEDs) allows for higher peak brightness, better-saturated colors and improved device lifetimes. ${ }^{8-10}$ Their practical use arises from the huge amount of work spent in colloidal synthesis and in understanding their chemical and physical properties. The amount of knowledge acquired on these systems will further boost the development and optimization of emerging novel nanostructures. ${ }^{11-12}$

Among the numerous topics in QDs photophysics, charge trapping is one of the most debated. ${ }^{13-14}$ The unsaturated bonds, located on the QDs surface, act as charge acceptor sites, promoting both exciton dissociation and charged QDs formation. Namely, the presence of surface charges deeply affects the exciton dynamics and originates a number of intriguing effects such as photoluminescence (PL) blinking and Auger-enhanced charge transfer (AE-CT) ${ }^{15-16}$. PL blinking or intermittency is a random alternation of bright and dark periods in QDs emission under continuous illumination. ${ }^{17-18}$ Although its mechanism is still unclear, many evidences relate it to charge trapping. ${ }^{19-20}$ Furthermore, as recently discovered, the enhancement of Auger interactions in surface charged QDs causes non-Marcus behavior in charge-transfer processes. ${ }^{15,}$ 21-22 Depending on the application, all these photo-physical processes can be either useful or detrimental. A rational development of nanostructures, with tailored trapping, is crucial in view of their practical application. 
To this aim, the introduction of different inorganic core/shell nanostructures paved the way to a wavefunction engineering approach. ${ }^{23-24}$ By acting on the chemical composition, size and shape parameters, the presence of an inorganic shell modifies not only the number but also the spatial and energetic distribution of traps, eventually altering their interaction with excitons generated in the core of QD. Inevitably, this further complicates the photo-physical picture of charge trapping. Only few works have hitherto focused on the interplay between charge trapping at the different interfaces and their effect on QDs optical properties. ${ }^{25-27}$ Recently, our group reported on the effect of trapping on PL dynamics in core/shell CdSe/Cd $\mathrm{Zn}_{1-\mathrm{x}} \mathrm{S}$ QDs. ${ }^{28}$ We interpreted transient PL (TRPL) data formulating a phenomenological model, comprising the balance between charge trapping and de-trapping processes. The former involves the formation of surface or interface trapped charge states, whereas de-trapping regenerates bound exciton states. The delayed generation of emissive bound exciton states well accounts for the long $\left(>100\right.$ ns to microseconds $\left.{ }^{19}\right)$ timescale of the PL decay of core/shell QDs. As a noteworthy result, we demonstrated that an accurate fitting of the PL data requires two trap distributions. Such result is in line with other works on CdSe/ZnS QDs, in which a similar behavior has been reported. ${ }^{29}$ The presence of two energetic distributions of exciton traps can be related, respectively, to surface and interface in core/shell heterostructures. The ratio between surface and interface trapping rates suggested that, although the majority of traps are located on the surface, a considerable amount of trapping sites forms at the core/shell interface. Albeit the dynamics of these processes is now understood, the energetics remains widely unexplored. The dispersion of reported PLQY values for QDs, measured under different excitation energies, demonstrates the need to fill this knowledge gap. The broadband absorber nature of QDs allows excitation over the entire visible spectrum, however the emission efficiency of these materials depends on excitation conditions and decreases at increasing excitation energy. This is due to a higher probability for accessing non-emissive trap states when the excitation energy is increased above the energy gap. ${ }^{31}$ Such peculiarity cannot be disregarded as, for instance, both electrically and optically pumped QD-LED work under high-energy excitation. 
Recently, Kambhampati investigated the existence of PLQY excitation spectra ${ }^{30}$, namely the dependence of PLQY on the photon energy used for excitation, by microscopic modeling of QDs temperature dependent PL spectra. ${ }^{31-32}$ A previously reported work on the competition between relaxation of band edge excitons and trapping at surface defects of small CdSe dots was extended to account for the dynamics of "hot excitons" generated by high energy photons. ${ }^{13}$ By means of a semi-classical electron transfer model, Kambhampati related the excitation energy dependence (EED) of PLQY with energy-dependent trapping rates in core CdSe QDs. ${ }^{30}$ The outcome of that analysis led to the prediction that the PLQY may exhibit a dependence on the excitation energy that markedly increases on lowering the temperature. Kambhampati's analysis was based on data from ultrafast time resolved measurements. Direct experimental measurements of the excitation dependence of steady-state PL were carried out either by observing deviations of PL excitation spectra from absorption spectra or by directly measuring the PLQY. Nevertheless, the variable and conflicting nature of experimental reports on PLQY excitation spectra suffered from variance in synthesis and passivation quality. ${ }^{33-36}$

The aim of this work is to extend the investigation of the EED of PLQY and of the underlying trapping and de-trapping mechanisms to core/shell CdSe QDs with different composition and structure of the shell. This will offer the opportunity to analyze how surface/interface trapping changes when structure and energy gap of the shell are changed while keeping constant the core properties. A study on well-defined systems, such as core/shell QDs, offers the possibility to get physical insights of broad significance. In addition to this, we also discuss why steady-state measurements are likely to yield results different from those inferred from ultrafast time resolved measurements. The key factor here is the effect of long-lived trapped charges on further photoexcitation and enhanced Auger recombination.

Specifically, here we report on the excitation energy dependence of steady-state PL for three series of $\mathrm{CdSe} / \mathrm{Cd}_{\mathrm{x}} \mathrm{Zn}_{1-\mathrm{x}} \mathrm{S}$ graded core/shell QDs. We probed the influence of charge trapping processes by measuring the photo-luminescence quantum yield (PLQY) under different excitation 
wavelengths. We observed a global increase of PLQY with decreasing excitation energy in the 2.25-3.10 eV range (550-400 nm wavelengths), together with a dependence on the shell thickness. Finally, we discuss and compare these steady-state results with the outcomes of the abovementioned charge trapping phenomenological kinetic model. ${ }^{28}$ Notably, we found that the exciton de-trapping plays a pivotal role also in determining the steady-state optical properties. Within this framework, we interpret the excitation wavelength dependence of PLQY and the mismatch between the measured PLQY and the estimated one as a steady-state signature of "hot-carrier" trapping. ${ }^{13,30-}$

31 The resulting photo-physical picture bridges energetics and dynamics of carrier trapping and highlights the role of different core/shell interfaces in determining PL properties. Furthermore, the investigation of different core-shell systems highlights the technological relevance of the different interfaces.

\section{Methods}

QDs synthesis

For the synthesis of QDs, we referred to previously published procedures. CdSe cores were prepared following a slightly modified method described by van Embden et al. ${ }^{37}$ Core-shell quantum dots were prepared according to a modified SILAR protocol that has been previously published. ${ }^{38}$

\section{Optical Characterization and PLQY Measurements}

We recorded UV-Vis spectra by using a Cary 5 spectrometer (Varian), in the range 200-800 nm. The PL spectra were measured with a FluoroMax-P (Jobin-Yvon) fluorimeter. All spectra were recorded from QDs colloidal solutions in chloroform, using $1 \mathrm{~cm}$ square quartz cuvettes. For spectrally-resolved PLQY measurements we adopted a relative technique. We calculated relative PLQY values following the Demas and Crosby procedure ${ }^{39}$, using different fluorescence standards 
for different excitation energies. Namely, we used Coumarin 153 (FQY=55\%), Rhodamine6G $(\mathrm{FQY}=93 \%$ ) and Cresyl Violet $(\mathrm{FQY}=66 \%)$ for the $3 \mathrm{eV}, 2.5 \mathrm{eV}$ and $2.2 \mathrm{eV}$ region, respectively.

We calculated quantum yield using the equation: $Q Y_{x}=Q Y_{r e f}\left(\frac{A_{r e f}\left(\lambda_{r e f}\right)}{A_{x}\left(\lambda_{x}\right)}\right)\left(\frac{I\left(\lambda_{r e f}\right)}{I\left(\lambda_{x}\right)}\right)\left(\frac{D_{x}}{D_{r e f}}\right)\left(\frac{n_{x}}{n_{r e f}}\right)^{2}$

where $I(\lambda)$ is the relative intensity of the exciting light at wavelength $\lambda, n$ is the refractive index of the solution, $D$ is the integrated area under the scattering-corrected emission spectrum and $A(\lambda)$ is the absorbance of the solution at the exciting wavelength $\lambda$.

In order to minimize errors, we adopted some cautions. First of all, we used a maximum absorbance of 0.15 for QDs solutions, in order to minimize the reabsorption effects. Secondly, we used Lambert-Beer correction to keep into account reabsorption effects on both excitation and emission photons.

\section{Results and Discussion}

Three different series of $\mathrm{CdSe} / \mathrm{Cd}_{\mathrm{x}} \mathrm{Zn}_{1-\mathrm{x}} \mathrm{S}$ QDs were investigated in this work, $\mathrm{CdS}$ and $\mathrm{Cd}_{0.5} \mathrm{Zn}_{0.5} \mathrm{~S}$ (henceforth $\mathrm{CdZnS}$ ) shells were epitaxially grown on $3.8 \mathrm{~nm}$ CdSe core QDs, with shell thicknesses ranging from 1 to 6 monolayers (ML). Homogeneous $\mathrm{ZnS}$ shells, with thickness ranging from 1 to $5 \mathrm{ML}$ were grown on $4.2 \mathrm{~nm} \mathrm{CdSe}$ core QDs. We aided the challenging epitaxial growth of $\mathrm{ZnS}$ on $\mathrm{CdSe}$, due to a $12 \%$ lattice mismatch, ${ }^{40}$ by interposing an enriching layer of CdS, whose lattice mismatch is about three times lower. ${ }^{41}$ More details on the synthesis procedure of CdSe cores and shell growth by successive ionic layer adsorption and reaction (SILAR) technique are reported in previous papers. ${ }^{24,37-38}$ For characterization by high resolution electron microscopy we refer to our previous work. ${ }^{42}$

Linear Characterization. In Figure 1A-C we report the evolution of the linear absorption spectrum of the three series ( 1 to $6 \mathrm{ML}, 1$ to $5 \mathrm{ML}$ for $\mathrm{CdSe} / \mathrm{ZnS}$ ) in solution. Absorption spectra of CdSe are described in terms of their excitonic structure. ${ }^{43-44}$ The band edge exciton (|1S> exciton), 
is related to $1 \mathrm{~S}_{\mathrm{e}}-1 \mathrm{~S}_{3 / 2}$ electron-hole pair creation. Transitions at higher energy $(|1 \mathrm{P}>| ,2 \mathrm{~S}>, \ldots)$ are related to the excitation of more energetic electron-hole pairs. ${ }^{45}$ Above $2.75 \mathrm{eV}$, the high joint density of states results in a broadened band, which is related to the excitation of so-called "hot carriers" (i.e. unbound electron-hole pairs).
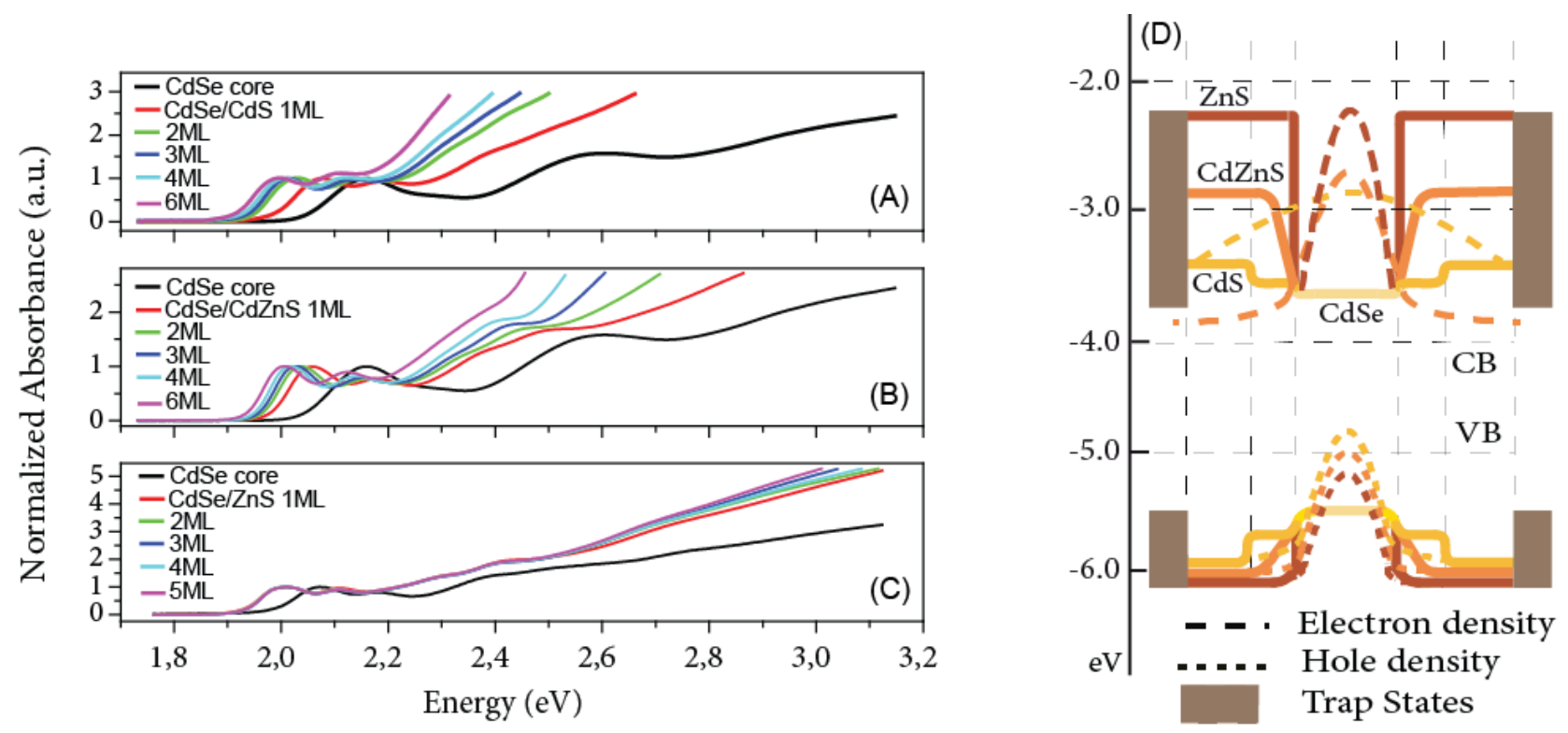

Figure 1- Normalized (at the |1S> exciton peak absorption) linear absorption spectra of (A) CdSe/CdS series (1-6 ML), (B) CdSe/CdZnS series (1-6 ML), (C) CdSe/ZnS series (1-5 ML) in solution. (D) Scheme of energy levels and electron/hole wavefunctions in different core/shell heterostructures. The dashed curves symbolize the wavefunction distribution and do not refer to the energy scale on the left. The spill out of the exciton wavefunction gradually decreases with increasing Zn concentration.

For the $\mathrm{CdSe} / \mathrm{CdS}$ series (Fig. 1A) the increase in shell thickness results in a redshift of the excitonic bands $(\approx 150 \mathrm{meV}$ for $6 \mathrm{ML})$. A similar behavior, though less pronounced, is observed for $\mathrm{CdSe} / \mathrm{CdZnS}$ series, where the redshift for the thickest shell is up to $120 \mathrm{meV}$ (Fig. 1B). As already pointed out before, this phenomenon is related to the spill out of the exciton wavefunction into the surrounding shell. ${ }^{46-47}$ The delocalization of the exciton wavefunction over the shell volume results in a reduced confinement effect and a consequent redshift due to a reduced quantum size effect. Consistently, CdSe/CdS is a "quasi-Type II" hetero-structure, ${ }^{48}$ where the electron wavefunction is 
completely delocalized in the QD volume whereas the hole is confined in the core. The increase in $\mathrm{Zn}$ anion concentration progressively creates a steeper potential barrier for the electronic motion and increase the "type-I" 41 nature of the core-shell hetero-structure. For this reason, the CdSe/ZnS series reveals a substantial independence of exciton energies from shell thickness. A scheme representing energy levels and wave functions in different core-shell heterostructures is reported in Figure 1D. Notably, in an ideal "type-I" hetero-structure the shell confinement should restrain the band edge exciton wavefunction away from the outer surface trap sites, thereby reducing the trapping probability. However, the epitaxial growth of the shell can result in the formation of lattice defects at the core/shell interface. ${ }^{40}$ The outer surface uncoordinated atoms, as well as the undercoordinated sites and dislocations at core/shell interfaces can act as traps for excitons. In 2005 Mews identified the effect of interface defects on the optical properties of core/shell QDs. ${ }^{41} \mathrm{He}$ used PLQY as a suitable parameter to quantify non-radiative relaxation pathways and evaluate their relative importance. Both interface and surface defects (i.e. dislocation and dangling bonds, respectively) are expected to affect PLQY.

In SI (Fig. SI1) we report narrow PL bands for the three QD series: the FWHM of PL band is $\approx 70$ $\mathrm{meV}$ for the $\mathrm{CdSe} / \mathrm{CdS}(\mathrm{SI})$ and $\mathrm{CdSe} / \mathrm{CdZnS}$ series and drops to $\approx 60 \mathrm{meV}$ for the $\mathrm{CdSe} / \mathrm{ZnS}$ series (SI). The observed Stokes shift $(\approx 10 \mathrm{meV})$ is consistent with commonly reported values. This PL band originates from the radiative relaxation of the $\mid 1 \mathrm{~S}>$ exciton. In all cases, we did not observe any trapped exciton emission band ${ }^{26}$ under $2.25 \mathrm{eV}$ excitation. However, despite the absence of a surface defect emission band, trapping processes cannot be neglected when describing the PL properties of QDs. ${ }^{49}$ In addition, effects such as interface defects must be considered, albeit the increasing confinement exerted by the shell should lead to higher PLQY. 


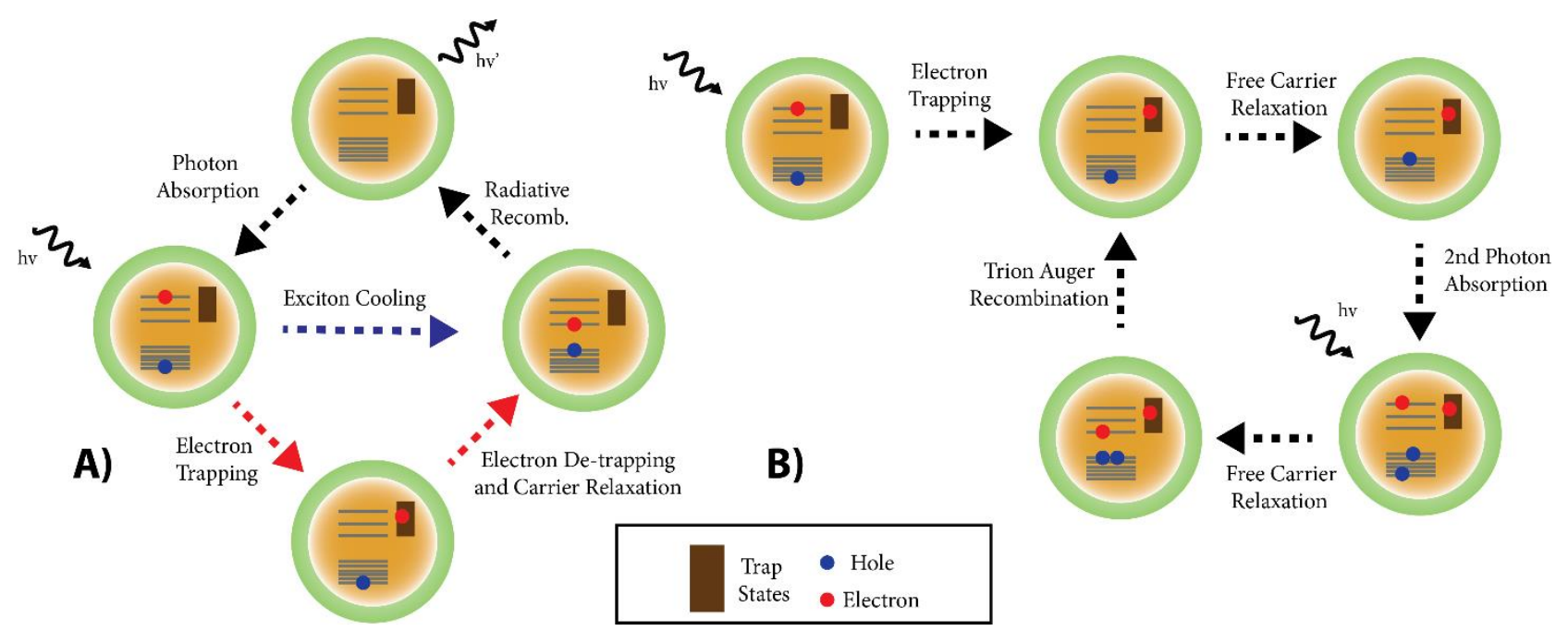

Figure 2 - Scheme representing the evolution of excited states in core/shell QDs in the presence of carrier trapping, during a steady-state spectroscopy experiment. Two photo-physical scenarios can be envisaged, depending on the presence of carrier de-trapping. (A) After the first photo-excitation, exciton cooling process competes with fast carrier trapping. Exciton cooling results in cold exciton species, able to undergo radiative relaxation. Carrier trapping, on the other hand, localizes a charge carrier at surface/core-shell interface. The restoring of bound exciton species, via carrier de-trapping process, allows radiative recombination to take place. (B) A different scenario comprising carrier trapping, after the first photo-excitation. A free charge can also undergo cooling (through ligand vibration coupling) and a long-living charge separated state is formed. The subsequent photo-excitation generates trion species, which rapidly recombine via Auger recombination. This second pathway, due to the presence of long-living localized charges, gives a negligible contribution to steady state PL. Accumulation of charges at surfaces generates a subset of dark QDs within the ensemble. Therefore, it is detrimental to PLQY.

PLQY Measurements and Modeling. The PLQY is a phenomenological parameter directly quantifying the conversion of absorbed photons into emitted photons. The measurement of this parameter usually relies on the spectrally integrated emission intensity in a steady-state experiment. During these measurements, QDs are repeatedly excited and the emission is detected on time-scales 
considerably longer than the exciton lifetime. The main processes that can occur during the photoexcitation of QDs are depicted in Figure 2.

According to the literature, ${ }^{25-26,29}$ we refer to carrier trapping as a charge-transfer process, resulting in localized charges whose excess energy is slowly and non-radiatively released via coupling to ligand vibrations. Nevertheless, the effect of carrier trapping on PLQY is twofold. Beside the first loss of photon conversion due to trapping, the long-living localized charges give also rise to photo-charged QDs. ${ }^{50}$ Photo-charged species strongly affect the recombination mechanism of subsequent excitations during the experiment, promoting non-radiative Auger recombination (recently also defined as "trion Auger recombination"51-52). This second aspect, given the microsecond to millisecond reported charge lifetimes, is potentially the most detrimental to the PL efficiency. An estimate of the likelihood that a charged QD absorbs a second photon during the PLQY measurements and follows the path of Fig. 2B is given in the SI.

The above considerations rely on a static view of trapped charges, acting as sources for nonradiative relaxation pathways. We and others ${ }^{25,} 28-29$ recently contributed to demonstrate a dynamical nature of trapped charges. A de-trapping process (Figure 2A) constitutes an essential part of the phenomenological model explaining the QDs PL dynamics. The importance of de-trapping rates in determining PLQY becomes even clearer when we compare the PLQY with the ratio ( $\left.\mathrm{R}_{\mathrm{DT}}\right)$ between de-trapping and trapping rate constants obtained from the fitting of the PL data of Ref. 28 according to a refined phenomenological model (See the SI). The fraction of bright QDs that determines the PL QY of a QD ensemble in steady-state depends on the ratio of detrapping/trapping rates, $v_{d} / v_{t}$. Under steady-state conditions, $\frac{v_{d}}{v_{t}} \propto \frac{k_{d}}{k_{t}}=R_{D T}$. The proportionality constant (see the SI) may show some variation on increasing the number of shell monolayers due to changes in the ensemble averaged densities of empty and charged traps. Apart from this, the PL QY is expected to correlate with $R_{D T}$. 
As shown in Figure 3, PLQY and $\mathrm{R}_{\mathrm{DT}}$ exhibit an analogous trend with respect to shell thickness, i.e. for all the QDs series an increase of $\mathrm{R}_{\mathrm{DT}}$ corresponds to an increase of the PLQY. Keep in mind, however, that the two parameters, $\mathrm{R}_{\mathrm{DT}}$ and the PLQY, are plotted on different scales and the comparison of the two indicates that $\mathrm{R}_{\mathrm{DT}}$ acts to modulate the PLQY, not to determine its absolute value. This suggests that whenever the de-trapping rate decreases with respect to the trapping rate the non-radiative rate increases. Although this result might seem trivial, it is important to emphasize that we measured the PLQY values from steady-state PL experiments and thereby independently from $\mathrm{R}_{\mathrm{DT}}$, which we extracted by fitting the PL decays with the phenomenological model. The direct relation between these two independently obtained parameters is a proof of the detrimental effect of accumulated charges on PLQY.

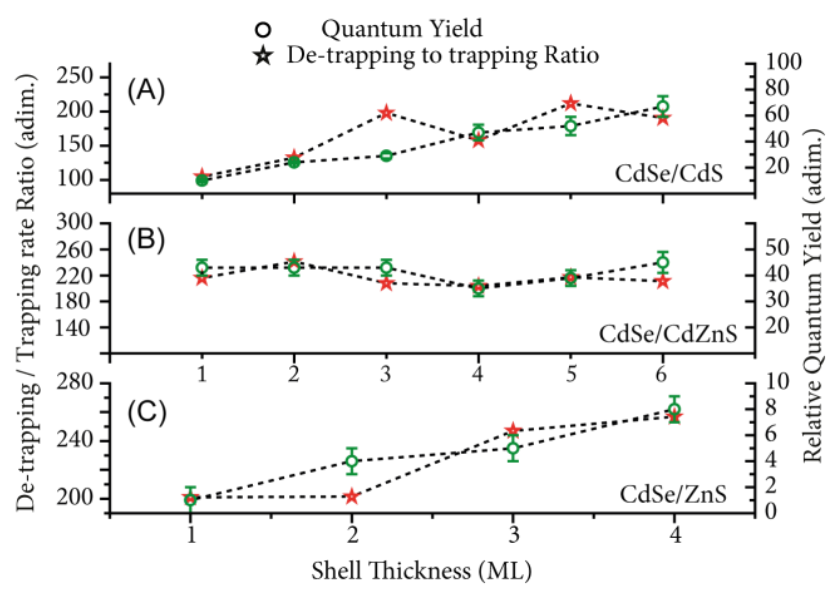

Figure 3 - Direct comparison between the de-trapping/trapping rate constants ratio (obtained from phenomenological model fitting of TRPL data using a 3.1eV excitation) and measured PLQY (by steady state PL experiments using a $2.53 \mathrm{eV}$ excitation). The comparison is extended to each sample of the three series (A) CdSe/ZnS, (B) CdSe/CdZnS, (C) CdSe/CdS. Data on the rate coefficients and $R_{D T}$ values are given in the SI.

The importance of the exciton de-trapping processes in influencing steady-state properties is dependent on the energy distribution of the trap states. Excitations at energies higher than the bandgap generate "hot" excitons with different carrier distribution, that are subject to charge transfer 
with fast rates to high-lying trap levels (HTs). Such HTs contain several quanta of ligand vibrations and the trapped charges are formed under non-equilibrium conditions from which they relax and become long lived.

To investigate these processes, we performed excitation wavelength resolved PLQY measurements on the three $\mathrm{CdSe} / \mathrm{Cd}_{\mathrm{x}} \mathrm{Zn}_{1-\mathrm{x}} \mathrm{S}$ QDs series by exciting them at three different energies: 2.25, 2.53 and $3.10 \mathrm{eV}$. As shown in Figure 4, PLQY values are strongly dependent on the excitation energy. We observe up to an eight-fold increased PLQY by lowering the excitation energy from $3.1 \mathrm{eV}$ to $2.25 \mathrm{eV}$. Loomis ${ }^{35}$ reported similar observations, by considering the mismatch between absorption and excitation profiles in core and core/shell quantum dots. A complementary explanation of the observed phenomena should consider, beside the energy distribution of exciton traps, also the relation between trapping and de-trapping rates and PLQY.

In our experiments, we span across a $1 \mathrm{eV}$ excitation energy interval and the highest excitation energy used lies about $1.2 \mathrm{eV}$ above the CdSe band-gap. Highly energetic photons generate "hotcarriers", whose wavefunction distribution is different from and carries higher energy than the one of bound excitons. Hot-carriers were recently observed to undergo ultrafast trapping at HT levels. ${ }^{13}$ Under continuous irradiation, charges accumulate at HT levels, increasing the non-radiative Auger recombination (Figure 2) and reducing the beneficial recovery of bound excitons via de-trapping. Therefore, the HT harmfulness stems from their higher trapping rate compared to the de-trapping rate, thus generating long lived trapped charges. The accumulation of charges at HT levels explains, according to the scheme in Figure 2, the drastic changes in PLQY values that cannot be justified starting from the trapping and de-trapping rates extracted from TRPL measurements. ${ }^{28}$ Microscopically, this is consistent with theoretical predictions relating the excitation energy dependence of PLQY to energy dependent carrier trapping rates. ${ }^{30-31}$

In addition to this, the dependence of PLQY on excitation energy and shell thickness provides further information on the nature of these traps. All our measurements have been performed at room temperature and only changing the shell thickness within a core/shell series while maintaining 
the same CdSe core dimension. Therefore, within a Marcus-Jortner scheme for transfer of charges to traps, the relevant changes concern the electronic coupling ${ }^{53}$. In turn, this coupling is affected by the changes in the shape and amplitude of the confining potential and by the correlated changes in the carrier wavefunctions.

In the $\mathrm{CdSe} / \mathrm{CdS}$ and $\mathrm{CdSe} / \mathrm{CdZnS}$ series we observe a marked dependence on excitation wavelength, especially for thicker shells. On the other hand, for less than 3ML thick shells we report only a slight excitation wavelength dependence of PLQY. Incidentally, the Raman characterization of analogous series evidenced a peculiar behavior above a $3 \mathrm{ML}$ shell thickness. ${ }^{42}$ For the $\mathrm{CdSe} / \mathrm{CdS}$ we ascribed this to the formation of a $3 \mathrm{ML}$ thick $\mathrm{CdSeS}$ alloy at the core/shell interface, whereas for the $\mathrm{CdSe} / \mathrm{CdZnS}$ we did not observe alloy formation and inferred that an abrupt interface is formed when $\mathrm{CdZnS}$ is grown onto CdSe QDs.
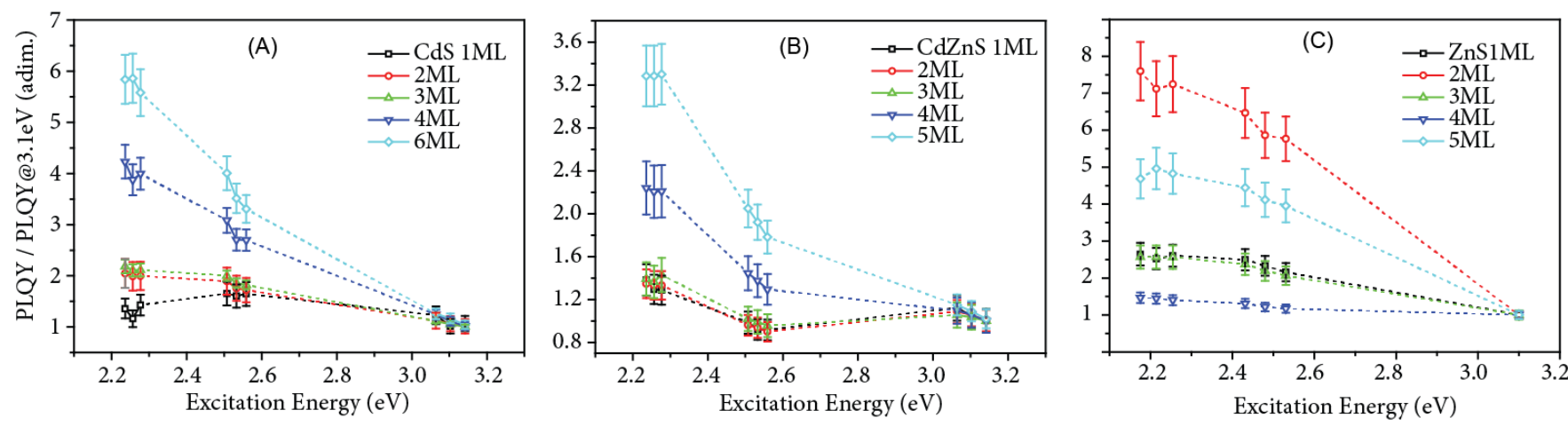

Figure 4 - Behavior of PLQY with respect to excitation wavelength. Data are reported for each sample of the three series $C d S e / C d S(A), C d S e / C d Z n S(B), C d S e / Z n S(C)$. Normalized PLQY values, with respect to PLQY measured under $3.1 \mathrm{eV}$ excitation, are reported. Each point indicates the relative increase of $P L Q Y$ with decreasing excitation energy from $3.1 \mathrm{eV}$ to $2.25 \mathrm{eV}$. Normalization values and the entire set of PLQY is reported in SI. Error bars are obtained propagating PLQY errors in Table SI_2. 
As shown in Figure 4, the smoother interface due to the alloy between the CdSe core and CdS shell allows a more efficient improvement of the PLQY as the excitation energy is lowered compared to the CdZnS series. The achievement of higher PLQYs in the presence of an alloyed core/shell interface, resulting in a smooth variation of the confinement potential, is in complete agreement with previously theoretical and experimental works by Efros and Klimov groups on the suppression of Auger recombination. ${ }^{48,54} \mathrm{We}$, and others, already reported this result in previous papers. By exciting at $3.1 \mathrm{eV}$ "hot-excitons" are generated both in the CdSe and in the CdS (energy gap is $2.42 \mathrm{eV}$ ) or CdZnS (energy gap $3 \mathrm{eV}$ from Vegard's law) shell, thereby the PLQY is lowered by the strong interaction with the outer surface HT states. At lower excitation energies, well below the gap of the shell material, hot-excitons are generated inside the CdSe core, leading to an increase of the PLQY for both CdS and CdZnS series (Figure 4A and 4B). Although the higher confining potential of CdZnS should minimize the interaction with surface HT sites, the increase of PLQY is more pronounced for CdSe/CdS QDs, especially for thick shell samples. At 6ML the increase of PLQY from $3.1 \mathrm{eV}$ to $2.25 \mathrm{eV}$ is only threefold for $\mathrm{CdZnS}$, whereas it is sixfold for CdS. This tells us that, in the case of CdZnS, part of the HT sites are located at the core/shell abrupt interface, from which they interact more easily with the core confined bound excitons. This result also suggests that interface HTs are distributed at a lower average energy compared to the outer surface HTs. On the other hand, for CdS the majority of the HT states resides in the outer shell, thanks to the smoother lattice parameter variation induced by the CdSeS alloy, which limits the formation of defects at the core-shell interface.

The relation between interfacial effects and PLQY excitation wavelength dependence is made even clearer by the $\mathrm{CdSe} / \mathrm{ZnS}$ series. None of the excitation energies used in this work exceeds the $>3.5 \mathrm{eV}$ band-gap of $\mathrm{ZnS}$, which means that excitons in this case are generated only in the CdSe core. For samples with one and two MLs of ZnS shell the PLQY increases when the excitation energy is reduced. In particular, for the $\mathrm{ZnS} 2 \mathrm{ML}$ sample we observe the steepest (eight-fold) PLQY recovery reducing from $3.1 \mathrm{eV}$ to $2.25 \mathrm{eV}$ excitation energy, mainly due to the efficient 
screening from the outer surface HT sites provided by the $\mathrm{ZnS}$ confinement potential. Furthermore, the CdS "enriching" layer deposited on top of the CdSe core prior to $\mathrm{ZnS}$ shell deposition limits the formation of traps at the core/shell interface. At shell thickness above $3 \mathrm{ML}$ the beneficial effect of the $\mathrm{ZnS}$ confinement potential is counteracted by the breakdown of the $\mathrm{ZnS}$ epitaxial growth and the formation of lattice dislocations. This increases the probability of interaction between "hotexcitons" and HTs, eventually leading to lower PLQYs absolute values (See the SI, Table SI_2) and reduces the PLQY recovery at lower excitation energy compared to samples with thinner shells.

\section{Conclusions}

To summarize, in this work we analyzed the drastic changes of steady-state PL properties under different excitation wavelengths. We observed decreasing PLQY values with increasing excitation energy, by factors reaching eight for the $\mathrm{CdSe} / \mathrm{ZnS}$ series, and distinct behavior with different chemical composition of the shell. We explained these findings in terms of charge trapping dynamics. By using the information obtained by applying a kinetic model to the analysis of TRPL data, ${ }^{28}$ we identified a correlation between the trapping and de-trapping rates and the PL efficiency. We then related the PLQY decrease at higher excitation energies to the fast trapping of hot carriers into vibrationally excited traps. The increased trapping/de-trapping ratio is unfavorable to the regeneration of emissive excitons. Instead, accumulation of trapped charges leads to loss of PLQY due to Auger trion recombination.

The different behavior of the EED of PLQY in the three series of core/shell QDs is explained as the result of differences in the confining potential and in the nature of the traps. A thorough characterization of the photophysical dynamics in the series of QDs investigated here via time resolved measurements is presently underway in our lab.

In the last few years there has been a fast growing interest in applications of semiconductor nanocrystals in lighting and display technologies, where QDs operate in steady-state conditions 
under highly energetic excitation or charge injection. The results presented here provide hints as to the choice of most appropriate shell structures for high efficiency emission.

\section{AUTHOR INFORMATION}

Corresponding Author: renato.bozio@unipd.it

A. M. is currently at Department of Physics and Astronomy and London Centre for Nanotechnology, University College London, Gower Street, London WC1E 6BT, United Kingdom

\section{ACKNOWLEDGMENT}

The authors acknowledge PRIN Prot.N. 2012T9XHH7 for financial support. J. Jaiseniak (Monash University, Australia) is gratefully acknowledged for providing us with CdSe core-shell quantum dots.

Supporting information Available: Photoluminescence spectra of core CdSe and core-shell $\mathrm{CdSe} / \mathrm{CdS}, \mathrm{CdSe} / \mathrm{CdZnS}$ and $\mathrm{CdSe} / \mathrm{ZnS}$. Excitation energy resolved PLQY values. This information are available free of charge via the Internet at http://pubs.acs.org.

\section{REFERENCES}

(1) Zhou, J.; Yang, Y.; Zhang, C.-y. Toward Biocompatible Semiconductor Quantum Dots: From Biosynthesis and Bioconjugation to Biomedical Application. Chemical Reviews 2015, 115, 11669-11717.

(2) Carey, G. H.; Abdelhady, A. L.; Ning, Z.; Thon, S. M.; Bakr, O. M.; Sargent, E. H. Colloidal Quantum Dot Solar Cells. Chemical Reviews 2015, 115, 12732-12763. 
(3) Klostranec, J. M.; Chan, W. C. W. Quantum Dots in Biological and Biomedical Research: Recent Progress and Present Challenges. Advanced Materials 2006, 18, 1953-1964.

(4) Freeman, R.; Finder, T.; Willner, I. Multiplexed Analysis of $\mathrm{Hg}^{2+}$ and $\mathrm{Ag}^{+}$Ions by Nucleic Acid Functionalized CdSe/ZnS Quantum Dots and Their Use for Logic Gate Operations. Angewandte Chemie International Edition 2009, 48, 7818-7821.

(5) Yuan, M.; Liu, M.; Sargent, E. H. Colloidal Quantum Dot Solids for Solution-Processed Solar Cells. Nature Energy 2016, 1, 16016.

(6) Claussen, J. C.; Hildebrandt, N.; Susumu, K.; Ancona, M. G.; Medintz, I. L. Complex Logic Functions Implemented with Quantum Dot Bionanophotonic Circuits. ACS Applied Materials \& Interfaces 2014, 6, 3771-3778.

(7) Bourzac, K. Quantum Dots Go on Display. Nature 2013, 493, 283-283.

(8) Kim, T.-H. et al., Full-Colour Quantum Dot Displays Fabricated by Transfer Printing. Nature Photonics 2011, 5, 176-182.

(9) Talapin, D. V.; Steckel, J. Quantum Dot Light-Emitting Devices. MRS Bulletin 2013, 38, 685-691.

(10) Luo, Z.; Xu, D.; Wu, S.-T. Emerging Quantum-Dots-Enhanced LCDs. J. Display Technol. 2014, 10, 526-539.

(11) Kamat, P. V.; Scholes, G. D. Quantum Dots Continue to Shine Brightly. The Journal of Physical Chemistry Letters 2016, 7, 584-585.

(12) Kim, J. Y.; Voznyy, O.; Zhitomirsky, D.; Sargent, E. H. $25^{\text {th }}$ Anniversary Article: Colloidal Quantum Dot Materials and Devices: A Quarter-Century of Advances. Advanced Materials 2013, $25,4986-5010$.

(13) Kambhampati, P. Hot Exciton Relaxation Dynamics in Semiconductor Quantum Dots: Radiationless Transitions on the Nanoscale. The Journal of Physical Chemistry C 2011, 115, 22089-22109. 
(14) Tyagi, P.; Kambhampati, P. False Multiple Exciton Recombination and Multiple Exciton Generation Signals in Semiconductor Quantum Dots Arise from Surface Charge Trapping. The Journal of Chemical Physics 2011, 134, 094706.

(15) Zhu, H.; Yang, Y.; Hyeon-Deuk, K.; Califano, M.; Song, N.; Wang, Y.; Zhang, W.; Prezhdo, O. V.; Lian, T. Auger-Assisted Electron Transfer from Photoexcited Semiconductor Quantum Dots. Nano Letters 2014, 14, 1263-1269.

(16) Mahler, B.; Spinicelli, P.; Buil, S.; Quelin, X.; Hermier, J.-P.; Dubertret, B. Towards nonBlinking Colloidal Quantum Dots. Nat Mater 2008, 7, 659-664.

(17) Zhang, A.; Dong, C.; Liu, H.; Ren, J. Blinking Behavior of CdSe/CdS Quantum Dots Controlled by Alkylthiols as Surface Trap Modifiers. The Journal of Physical Chemistry C 2013, $117,24592-24600$.

(18) Chen, O., et al. Compact High-Quality CdSe-CdS Core-Shell Nanocrystals with Narrow Emission Linewidths and Suppressed Blinking. Nature Materials 2013, 12, 445-451.

(19) Rabouw, F. T.; Kamp, M.; van Dijk-Moes, R. J. A.; Gamelin, D. R.; Koenderink, A. F.; Meijerink, A.; Vanmaekelbergh, D., Delayed Exciton Emission and Its Relation to Blinking in CdSe Quantum Dots. Nano Letters 2015, 15, 7718-7725.

(20) Krauss, T. D.; Peterson, J. J., Quantum Dots: A Charge for Blinking. Nature Materials 2012, $11,14-16$.

(21) Subha, R.; Nalla, V.; Lim, E. J. Q.; Vijayan, C.; Huang, B. B. S.; Chin, W. S.; Ji, W., Slow Down of Charge Transfer Owing to Auger Recombination and Two-Photon Action Cross-Section of CdS-CdSe-CdS Segmented Nanorods. ACS Photonics 2015, 2, 43-52.

(22) Olshansky, J. H.; Ding, T. X.; Lee, Y. V.; Leone, S. R.; Alivisatos, A. P., Hole Transfer from Photoexcited Quantum Dots: The Relationship between Driving Force and Rate. Journal of the American Chemical Society 2015, 137, 15567-15575. 
(23) Ning, Z.; Tian, H.; Qin, H.; Zhang, Q.; Ågren, H.; Sun, L.; Fu, Y. Wave-Function Engineering of $\mathrm{CdSe} / \mathrm{CdS}$ Core/Shell Quantum Dots for Enhanced Electron Transfer to a $\mathrm{TiO}_{2}$ Substrate. The Journal of Physical Chemistry C 2010, 114, 15184-15189.

(24) Fortunati, I.; Signorini, R.; Bozio, R.; Jasieniak, J. J.; Antonello, A.; Martucci, A.; Giustina, G. D.; Brusatin, G.; Guglielmi, M., CdSe Core-Shell Nanoparticles as Active Materials for UpConverted Emission. The Journal of Physical Chemistry C 2011, 115, 3840-3846.

(25) Jones, M.; Lo, S. S.; Scholes, G. D., Signatures of Exciton Dynamics and Carrier Trapping in the Time-Resolved Photoluminescence of Colloidal CdSe Nanocrystals. The Journal of Physical Chemistry C 2009, 113, 18632-18642.

(26) Mooney, J.; Krause, M. M.; Saari, J. I.; Kambhampati, P. Challenge to the Deep-Trap Model of the Surface in Semiconductor Nanocrystals. Physical Review B 2013, 87, 081201.

(27) Kern, S. J.; Sahu, K.; Berg, M. A. Heterogeneity of the Electron-Trapping Kinetics in CdSe Nanoparticles. Nano Letters 2011, 11, 3493-3498.

(28) Minotto, A.; Todescato, F.; Fortunati, I.; Signorini, R.; Jasieniak, J. J.; Bozio, R. Role of Core-Shell Interfaces on Exciton Recombination in CdSe-Cd $\mathrm{Zn}_{1-\mathrm{x}} \mathrm{S}$ Quantum Dots. The Journal of Physical Chemistry C 2014, 118, 24117-24126.

(29) Jones, M.; Lo, S. S.; Scholes, G. D., Quantitative Modeling of the Role of Surface Traps in CdSe/CdS/ZnS Nanocrystal Photoluminescence Decay Dynamics. Proceedings of the National Academy of Sciences 2009, 106, 3011-3016.

(30) Mooney, J.; Krause, M. M.; Kambhampati, P. Connecting the Dots: The Kinetics and Thermodynamics of Hot, Cold, and Surface-Trapped Excitons in Semiconductor Nanocrystals. The Journal of Physical Chemistry C 2014, 118, 7730-7739.

(31) Kambhampati, P., On the Kinetics and Thermodynamics of Excitons at the Surface of Semiconductor Nanocrystals: Are there Surface Excitons? Chemical Physics 2015, 446, 92-107.

(32) Krause, M. M.; Kambhampati, P. Linking Surface Chemistry to Optical Properties of Semiconductor Nanocrystals. Physical Chemistry Chemical Physics 2015, 17, 18882-18894. 
(33) Rumbles, G.; Selmarten, D. C.; Ellingson, R. J.; Blackburn, J. L.; Yu, P.; Smith, B. B.; Mićić, O. I.; Nozik, A. J. Anomalies in the Linear Absorption, Transient Absorption, Photoluminescence and Photoluminescence Excitation Spectroscopies of Colloidal InP Quantum Dots. Journal of Photochemistry and Photobiology A: Chemistry 2001, 142, 187-195.

(34) Tonti, D.; van Mourik, F.; Chergui, M., On the Excitation Wavelength Dependence of the Luminescence Yield of Colloidal CdSe Quantum Dots. Nano Letters 2004, 4, 2483-2487.

(35) Hoy, J.; Morrison, P. J.; Steinberg, L. K.; Buhro, W. E.; Loomis, R. A. Excitation Energy Dependence of the Photoluminescence Quantum Yields of Core and Core/Shell Quantum Dots. The Journal of Physical Chemistry Letters 2013, 4, 2053-2060.

(36) Dias, E. A.; Grimes, A. F.; English, D. S.; Kambhampati, P. Single Dot Spectroscopy of Two-Color Quantum Dot/Quantum Shell Nanostructures. The Journal of Physical Chemistry C 2008, 112, 14229-14232.

(37) van Embden, J.; Mulvaney, P. Nucleation and Growth of CdSe Nanocrystals in a Binary Ligand System. Langmuir 2005, 21, 10226-10233.

(38) van Embden, J.; Jasieniak, J.; Mulvaney, P. Mapping the Optical Properties of CdSe/CdS Heterostructure Nanocrystals: The Effects of Core Size and Shell Thickness. Journal of the American Chemical Society 2009, 131, 14299-14309.

(39) Crosby, G. A.; Demas, J. N. Measurement of Photoluminescence Quantum Yields. Review. The Journal of Physical Chemistry 1971, 75, 991-1024.

(40) Dabbousi, B. O.; Rodriguez-Viejo, J.; Mikulec, F. V.; Heine, J. R.; Mattoussi, H.; Ober, R.; Jensen, K. F.; Bawendi, M. G., (CdSe)ZnS Core-Shell Quantum Dots: Synthesis and Characterization of a Size Series of Highly Luminescent Nanocrystallites. The Journal of Physical Chemistry B 1997, 101, 9463-9475.

(41) Xie, R.; Kolb, U.; Li, J.; Basché, T.; Mews, A. Synthesis and Characterization of Highly Luminescent $\mathrm{CdSe}-$ Core $\mathrm{CdS} / \mathrm{Zn}_{0.5} \mathrm{Cd}_{0.5} \mathrm{~S} / \mathrm{ZnS}$ Multishell Nanocrystals. Journal of the American Chemical Society 2005, 127, 7480-7488. 
(42) Todescato, F.; Minotto, A.; Signorini, R.; Jasieniak, J. J.; Bozio, R., Investigation into the Heterostructure Interface of CdSe-Based Core-Shell Quantum Dots Using Surface-Enhanced Raman Spectroscopy. ACS Nano 2013, 7, 6649-6657.

(43) Klimov, V. I. Spectral and Dynamical Properties of Multiexcitons in Semiconductor Nanocrystals. Annual Review of Physical Chemistry 2007, 58, 635-673.

(44) Nirmal, M.; Norris, D. J.; Kuno, M.; Bawendi, M. G.; Efros, A. L.; Rosen, M. Observation of the "Dark Exciton" in CdSe Quantum Dots. Physical Review Letters 1995, 75, 3728-3731.

(45) Efros, A. L.; Rosen, M.; Kuno, M.; Nirmal, M.; Norris, D. J.; Bawendi, M. Band-Edge Exciton in Quantum Dots of Semiconductors with a Degenerate Valence Band: Dark and Bright Exciton States. Physical Review B 1996, 54, 4843-4856.

(46) Li, J.; Wang, L.-W. First Principle Study of Core/Shell Structure Quantum Dots. Applied Physics Letters 2004, 84, 3648-3650.

(47) Kossut, J. Quantum Dots: Squeeze or stretch? Nature Materials 2009, 8, 8-9.

(48) García-Santamaría, F.; Chen, Y.; Vela, J.; Schaller, R. D.; Hollingsworth, J. A.; Klimov, V. I. Suppressed Auger Recombination in "Giant” Nanocrystals Boosts Optical Gain Performance. Nano Letters 2009, 9, 3482-3488.

(49) Kambhampati, P. Unraveling the Structure and Dynamics of Excitons in Semiconductor Quantum Dots. Accounts of Chemical Research 2011, 44, 1-13.

(50) McGuire, J. A.; Sykora, M.; Robel, I.; Padilha, L. A.; Joo, J.; Pietryga, J. M.; Klimov, V. I. Spectroscopic Signatures of Photocharging due to Hot-Carrier Transfer in Solutions of Semiconductor Nanocrystals under Low-Intensity Ultraviolet Excitation. ACS Nano 2010, 4, 60876097.

(51) Gong, K.; Kelley, D. F. Surface Charging and Trion Dynamics in CdSe-Based Core/Shell Quantum Dots. The Journal of Physical Chemistry C 2015, 119, 9637-9645.

(52) Vaxenburg, R.; Rodina, A.; Shabaev, A.; Lifshitz, E.; Efros, A. L. Nonradiative Auger Recombination in Semiconductor Nanocrystals. Nano Letters 2015, 15, 2092-2098. 
(53) Jortner, J. Temperature Dependent Activation Energy for Electron Transfer Between Biological Molecules. The Journal of Chemical Physics 1976, 64, 4860-4867.

(54) Cragg, G. E.; Efros, A. L. Suppression of Auger Processes in Confined Structures. Nano Letters 2010, 10, 313-317. 


\section{TOC GRAPHICS}

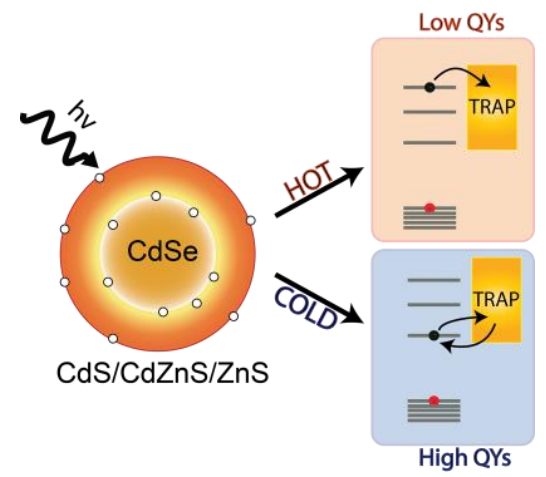

